Ferrata Storti Foundation

\title{
Targeting the endoplasmic reticulum- mitochondria interface sensitizes leukemia cells to cytostatics
}

Fabian Koczian, ${ }^{1}$ Olga Nagło, ${ }^{1}$ Jan Vomacka, ${ }^{2}$ Binje Vick, ${ }^{3}$ Phil Servatius, ${ }^{4}$ Themistoklis Zisis, ${ }^{1}$ Britta Hettich, ${ }^{1}$ Uli Kazmaier, ${ }^{4}$ Stephan A. Sieber, ${ }^{2}$ Irmela Jeremias, ${ }^{3}$ Stefan Zahler ${ }^{1}$ and Simone Braig ${ }^{1}$

${ }^{1}$ Department of Pharmaceutical Biology, Ludwig Maximilian University of Munich; ${ }^{2}$ Department of Chemistry, Technical University of Munich, Garching; ${ }^{3}$ Research Unit Gene Vectors, Helmholtz Zentrum München, German Center for Environmental Health, Munich and ${ }^{4}$ Institute of Organic Chemistry, Saarland University, Saarbrücken, Germany
Haematologica 2019

Volume 104(3):546-555

\section{Correspondence: \\ SIMONE BRAIG \\ simone.braig@cup.uni-muenchen.de \\ Received: May 9, 2018. \\ Accepted: October 4, 2018. \\ Pre-published: October 11, 2018.}

doi:10.3324/haematol.2018.197368

Check the online version for the most updated information on this article, online supplements, and information on authorship \& disclosures: www. haematologica.org/content/104/3/546

\section{(C)2019 Ferrata Storti Foundation}

Material published in Haematologica is covered by copyright. All rights are reserved to the Ferrata Storti Foundation. Use of published material is allowed under the following terms and conditions:

https://creativecommons.org/licenses/by-nc/4.0/legalcode. Copies of published material are allowed for personal or internal use. Sharing published material for non-commercial purposes is subject to the following conditions:

https://creativecommons.org/licenses/by-nc/4.0/legalcode, sect. 3. Reproducing and sharing published material for commercial purposes is not allowed without permission in writing from the publisher.

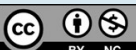

\section{ABSTRACT}

ombination chemotherapy has proven to be a favorable strategy to treat acute leukemia. However, the introduction of novel compounds remains challenging and is hindered by a lack of understanding of their mechanistic interactions with established drugs. In the present study, we demonstrate a highly increased response of various acute leukemia cell lines, drug-resistant cells and patient-derived xenograft cells by combining the recently introduced protein disulfide isomerase inhibitor PS89 with cytostatics. In leukemic cells, a proteomics-based target fishing approach revealed that PS89 affects a whole network of endoplasmic reticulum homeostasis proteins. We elucidate that the strong induction of apoptosis in combination with cytostatics is orchestrated by the PS89 target B-cell receptor-associated protein 31, which transduces apoptosis signals at the endoplasmic reticulum -mitochondria interface. Activation of caspase-8 and cleavage of B-cell receptor-associated protein 31 stimulate a pro-apoptotic crosstalk including release of calcium from the endoplasmic reticulum and an increase in the levels of reactive oxygen species resulting in amplification of mitochondrial apoptosis. The findings of this study promote PS89 as a novel chemosensitizing agent for the treatment of acute leukemia and uncovers that targeting the endoplasmic reticulum - mitochondrial network of cell death is a promising approach in combination therapy.

\section{Introduction}

Despite the significant success in the management of childhood acute lymphoblastic leukemia (ALL) and acute myeloid leukemia (AML) with survival rates of $>80 \%$ and $>60 \%$, respectively, ${ }^{1}$ the outcome of patients with relapsed or chemoresistant leukemia is still dismal. ${ }^{2,3}$ Especially in older patients, the balance of tolerable dosing versus effective cytotoxicity remains a major challenge. This issue is further exacerbated by the development of leukemic cell chemoresistance, which has been demonstrated for several cytostatics including tubulin binders and topoisomerase inhibitors. ${ }^{4,5}$ In addition, the emergence of relapse-specific mutations of cancer cells is often associated with resistance to thiopurines and glucocorticoids. ${ }^{6,7}$ Thus, novel pharmaceutical options are urgently needed for the improvement of current treatment regimens.

There is general consenius that combination therapies benefit from the crosstalk of antileukemic agents, however the mechanisms of interaction have only been explored for a few. ${ }^{8}$ Therefore, drug discovery is not only encouraged to identify novel compounds and targets, but also to enhance the understanding of their interdependence with established cytostatics. The concept of network pharmacology has raised great interest in recent years, especially regarding complex disease systems such as cancer., ${ }^{8,9}$ Following this principle, multi-target strategies, rather than the 'one drug, one target' paradigm, are proposed to be superior in rewiring cancer- 
specific networks and for overcoming the system robustness of cancer cell phenotypes. ${ }^{10,11}$ Translating this concept to combinatorial drug treatment, a highly interesting issue is not only how networks are locally perturbed by individual compounds, but moreover how interventions at multiple cellular loci cooperate. Considering potential proapoptotic target networks, the crucial role of the endoplasmic reticulum (ER)-mitochondria 'social network of cell death' was recently stressed in several studies highlighting the dynamic interaction of these two cellular elements. ${ }^{12,13}$ In this context, the B-cell receptor-associated protein 31 (BAP31) was described as a substrate of caspase- 8 and emerges as a communicator of apoptosis signals from the ER to mitochondria. ${ }^{14,15}$ Consistently, a role of the caspase 8-BAP31 axis has been demonstrated in ER stress-triggered apoptosis of B-cell lymphocytic leukemia cells. ${ }^{16}$ ER stress results from an imbalance between ER protein load and folding capacity. Protein disulfide isomerases (PDI) constitute a crucial family of enzymes for maintaining oxidative protein folding and ER homeostasis. ${ }^{17}$ Hence, these proteins have been recognized as exciting novel targets in cancer research. ${ }^{18}$ Furthermore, overexpression of PDI has been discovered in leukemia and linked to chemoresistance. ${ }^{19-21}$

Recently, we introduced the first reversible small-molecule PDI inhibitor PS89 which binds in close proximity to the catalytic centers of PDI. ${ }^{22}$ Moreover and contrary to other PDI inhibitors that are severely cytotoxic, ${ }^{23,24}$ PS89 is not toxic up to micromolar concentrations, although it has been shown to greatly enhance etoposide-induced apoptosis. This exceptional feature of effective chemosensitization at subtoxic doses motivated not only further combination therapy studies with PS89, but also a deeper analysis of its interactive signaling. In the present work, PS89 is set on stage as a novel therapeutic option for the treatment of acute leukemia. The favorable attributes of PS89 and its broad applicability are highlighted in ALL and AML cell lines, drug-resistant cells as well as patientderived xenograft (PDX) cells. The critical networks integrated in the synergistic pro-apoptotic signaling of PS89 in combination with cytostatics were identified, thus emphasizing the crucial function of ER-mitochondria communication for successful combination therapies.

\section{Methods}

\section{Cell cultures}

Jurkat cells (wild-type, CASP8-deficient, Bcl-2- and Bcl-xL-overexpressing) were kindly provided by P. H. Krammer (Heidelberg, Germany). CCRF-CEM and vincristine-resistant CEM cells ${ }^{25,26}$ were obtained from M. Kavallaris (Sydney, Australia), HEK 293 and HeLa cells from the Deutsche Sammlung von Mikroorganismen und Zellkulturen (DSMZ; Braunschweig, Germany) and HL-60 from the American Type Culture Collection (ATCC; Manassas, VA, USA). All cell lines were maintained in ATCC-recommended culture conditions.

\section{Patient-derived xenograft cells, peripheral blood mononuclear cells and CD34-positive cells}

The model of ALL and AML patients' leukemia cells growing in mice has been described previously. ${ }^{27,28}$ Ethical statements and approvals are outlined in the Online Supplementary Information. In the present study, PDX cells were freshly isolated from the bone marrow or spleen of NSG mice and cultured in the presence or absence of compounds. Peripheral blood mononuclear cells were freshly isolated from EDTA-anticoagulated blood of healthy donors by gradient centrifugation using Ficoll-Paque PLUS (GE Healthcare, Chicago, IL, USA) according to the manufacturer's instructions. The peripheral blood mononuclear cells were maintained in RPMI 1640 with $2 \mathrm{mM}$ glutamine supplemented with $20 \%(\mathrm{v} / \mathrm{v})$ fetal calf serum and $1 \mathrm{mM}$ pyruvate. CD34-positive cells were identified by staining with fluorescein isothiocyanate-conjugated anti-human CD34 antibody (BD Biosciences, Heidelberg, Germany) according to the manufacturer's instructions and analyzed by flow cytometry as described by Fukuda et al. ${ }^{29}$

\section{Ethical statements}

Written informed consent was obtained from all patients or legal guardians when the patients were minors. The study was performed in accordance with the ethical standards of the responsible committee on human experimentation (written approval from the ethics committee of the Ludwig Maximilian University Hospital, Munich, number 068-08) and with the Helsinki Declaration of 1975, as revised in 2000. All animal experiments were performed in accordance with the current ethical standards of the official committee on animal experimentation (written approval from Regierung von Oberbayern, number 55.2-1-542532-95-10).

\section{Activity-based protein profiling}

Jurkat cells were incubated with unmodified PS89 $(100 \mu \mathrm{M})$ or dimethyl sulfoxide (DMSO) as a control for $45 \mathrm{~min}$ at $37^{\circ} \mathrm{C}$ and in a second step with the PS89 photo probe $(20 \mu \mathrm{M})$ or DMSO as a control for $45 \mathrm{~min}$ at $37^{\circ} \mathrm{C}$. Cells were lysed in $1 \mathrm{~mL}$ phosphatebuffered saline with $1 \%(\mathrm{v} / \mathrm{v}) \mathrm{NP} 40$ and $1 \%$ (w/v) sodium deoxycholate and sonication for $15 \mathrm{~s}$ on ice. Sample preparation and mass spectrometry analysis of target proteins by gel-free activitybased protein profiling and dimethyl labeling was performed as previously described. ${ }^{30}$ Cutoff criteria for target identification were: (i) enrichment by photo probe $\log _{2}$ Probe/DMSO $>1.6,-\log _{10}$ $P$-value $>2$ and (ii) PS89 competition $\log _{2}$ Probe/PS89 $>0$. The data shown are the results of three biological replicates.

Details of the methods used are available in the Online Supplementary Appendix.

\section{Results}

PS89 sensitizes acute leukemia cell lines and patientderived xenograft cells to cytostatics

The concept of chemosensitization with the recently introduced PDI inhibitor PS89 was initially evaluated in a dose-response apoptosis assay. While PS89 was applied at a fixed subtoxic dose, the concentration of etoposide could be reduced by at least half to achieve equal cytotoxicity applying the combination treatment. This is in line with a more than 2 -fold shift in the half maximal effective concentration $\left(\mathrm{EC}_{50}\right)$ (Figure $\left.1 \mathrm{~A}\right)$. Bliss values indicating synergistic effects of PS89 in combination with etoposide are shown in Online Supplementary Table S1A. Whereas etoposide-treated cells showed a pronounced G2 arrest, PS89 had no effect on the cell cycle (Online Supplementary Figure S1A). PS89 treatment in combination with subtoxic concentrations of etoposide synergistically inhibited Jurkat cell proliferation and colony formation (Online Supplementary Figure $S 1 B, C)$. The ability of PS89 to induce synergistic apoptosis with diverse cytostatics could be translated to acute leukemia cells of different lineages. 
A

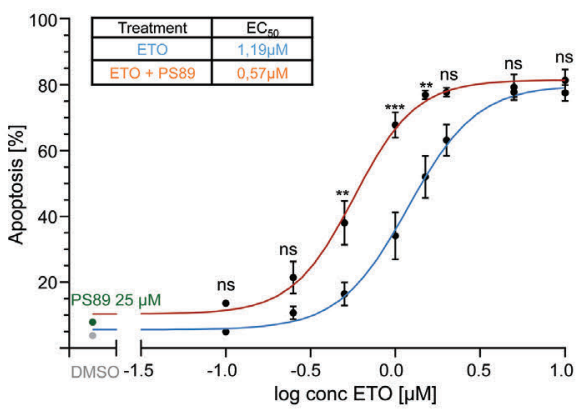

C

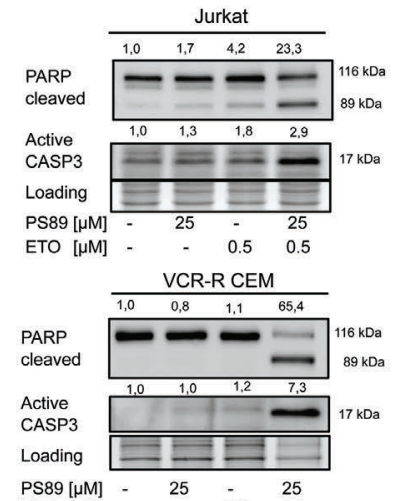

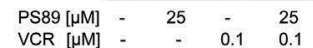

B

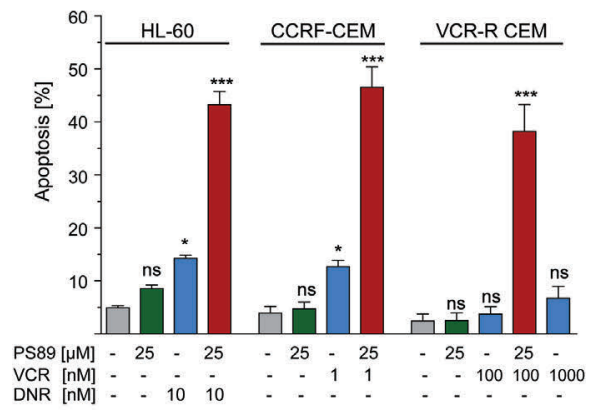

D

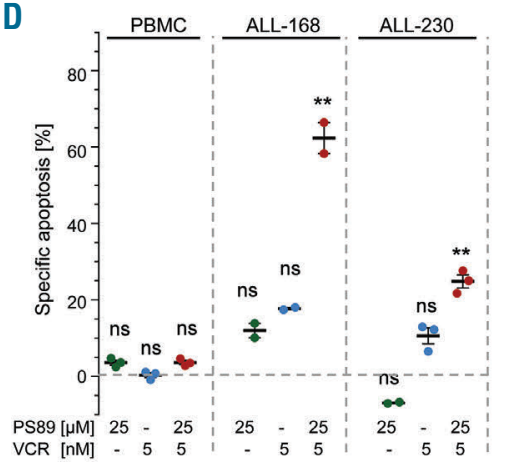

\begin{tabular}{|c|c|c|}
\hline $\begin{array}{c}\text { Bliss values } \\
(>1 \text { indicates } \\
\text { synergism) }\end{array}$ & $\begin{array}{c}\text { treatment } \\
5 \mathrm{nM} \mathrm{VCR} \\
\text { +PS89 }\end{array}$ & $\begin{array}{c}\text { synergistic } \\
\text { effect }\end{array}$ \\
\hline PBMC & 0.9 & no \\
\hline ALL-168 & 2.3 & yes \\
\hline ALL-230 & 5.6 & yes \\
\hline
\end{tabular}

E

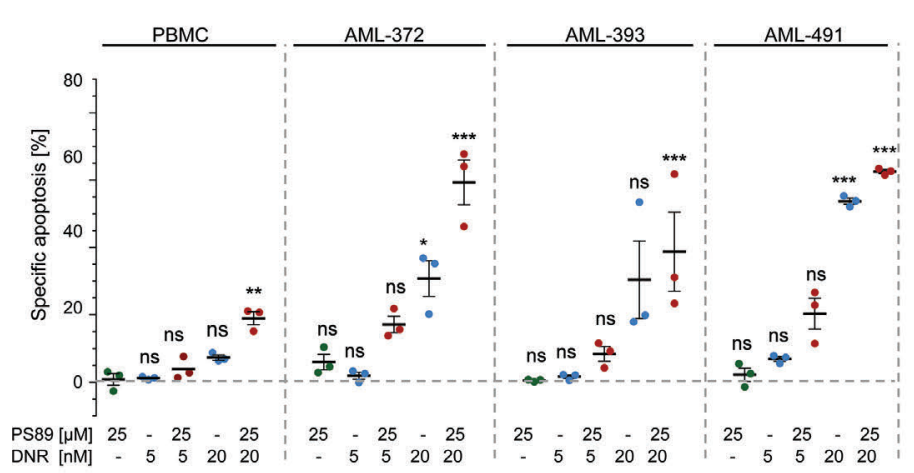

F

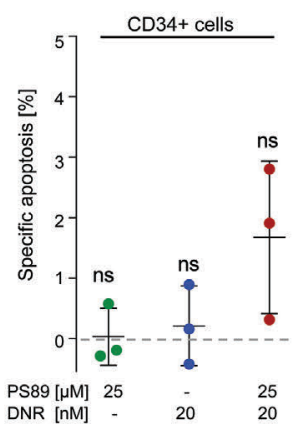

\begin{tabular}{|c|c|c|c|}
\hline $\begin{array}{c}\text { Bliss values } \\
(>1 \text { indicates } \\
\text { synergism) }\end{array}$ & $\begin{array}{c}\text { treatment } \\
\text { 5nM DNR } \\
\text { +PS89 }\end{array}$ & $\begin{array}{c}\text { treatment } \\
\text { 20nM DNR } \\
\text { +PS89 }\end{array}$ & $\begin{array}{c}\text { synergistic } \\
\text { effect }\end{array}$ \\
\hline PBMC & 2.0 & 2.4 & yes \\
\hline AML-372 & 2.2 & 1.7 & yes \\
\hline AML-393 & 3.9 & 1.3 & yes \\
\hline AML-491 & 2.3 & 1.1 & yes \\
\hline
\end{tabular}

Figure 1. Chemosensitization of acute leukemia cells with PS89. (A,B) Apoptosis of Jurkat, HL-60, CCRF-CEM and vincristine resistant (VCR-R) CEM cells treated with cytostatics (ETO: etoposide, VCR: vincristine, DNR: daunorubicin) in the presence or absence of $25 \mu \mathrm{M}$ PS89. The percentage of apoptotic cells was determined by FACS analysis after $48 \mathrm{~h}$. (C) Jurkat and VCR-R CEM were cultured for $48 \mathrm{~h}$ in drug-supplemented medium and apoptosis was analyzed by immunoblotting. (D,E) Freshly isolated peripheral blood mononuclear cells (PBMC) and acute lymphoblastic leukemia (ALL) or acute myeloid leukemia (AML) patient-derived xenograft cells were treated with PS89 and cytostatics for $48 \mathrm{~h}$ or $72 \mathrm{~h}$, respectively. Apoptotic cells were determined by FACS analysis and specific apoptosis was calculated in relation to untreated controls. Synergism was calculated using the Bliss independence model. (F) PBMC were treated for $48 \mathrm{~h}$ with the indicated drugs. CD34-positive cells were identified by flow cytometry using a fluorescein isothiocyanate-conjugated CD34 antibody. Cell death was analyzed by propidium iodide staining. 
This was demonstrated by combinations of PS89 with daunorubicin in HL-60 cells and vincristine in CCRF-CEM cells (Figure 1B, synergism calculations according to the Bliss independence model are shown in Online Supplementary Table S1B) as well as with 6-mercaptopurine and dexamethasone in Jurkat and HL-60 cells, respectively (Online Supplementary Figure S1D-F). Furthermore, vincristine-resistant CEM cells showed marked apoptosis in response to treatment with $100 \mathrm{nM}$ vincristine in combi- nation with PS89 (38.3\% apoptotic cells), while being resistant to 10 -fold higher vincristine concentrations without co-stimulation (1000 nM vincristine; 6.8\% apoptotic cells) (Figure 1B). Moreover, clonogenic growth of vincristine-resistant CEM and HL-60 cells was significantly abrogated upon treatment with PS89 in combination with vincristine or 6-mercaptopurine, respectively (Online Supplementary Figure $S 1 G, H)$. Following treatment of Jurkat, vincristine-resistant CEM, CCRF-CEM and HL-60
A
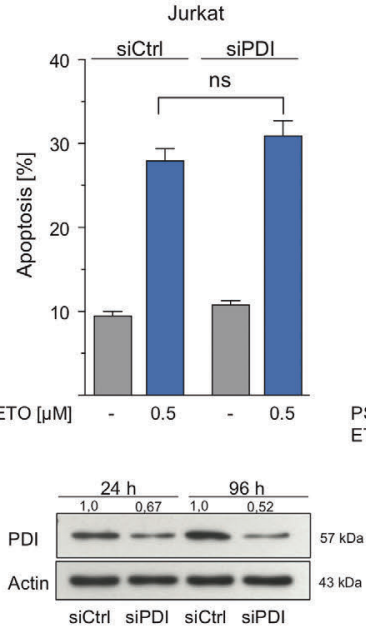

HEK 293

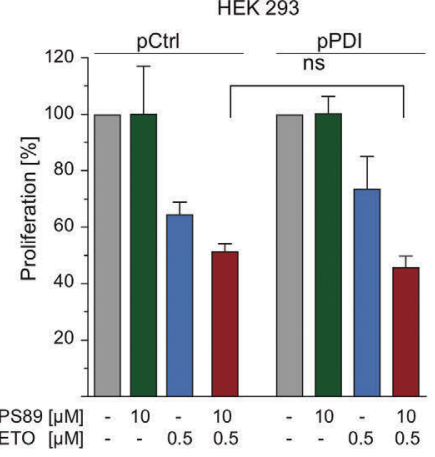

B
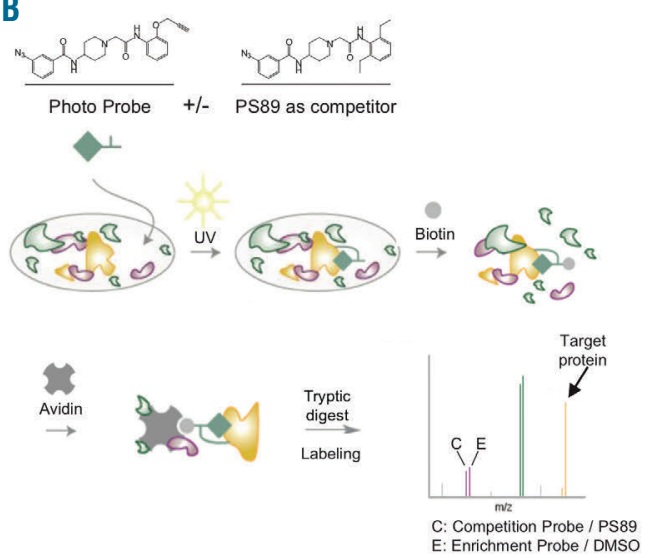

C: Competition Probe / PS89

C

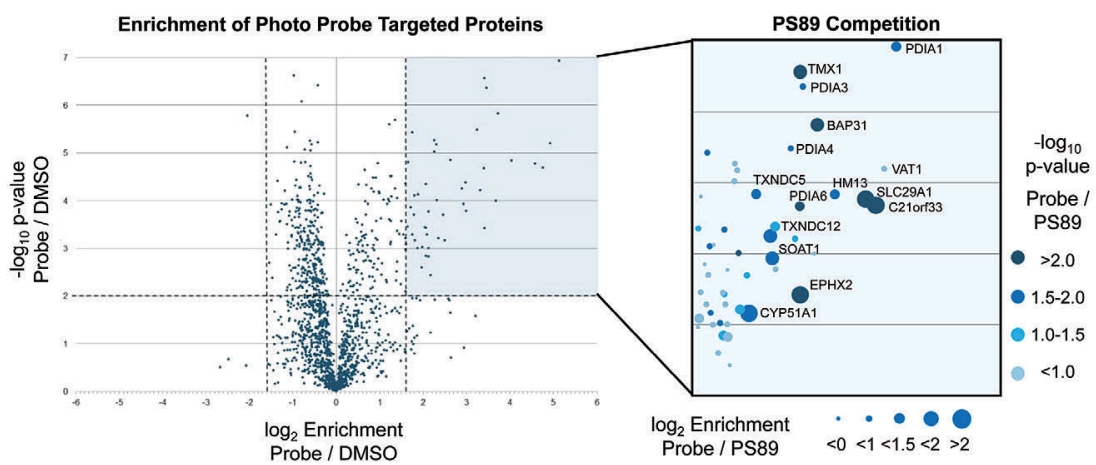

D

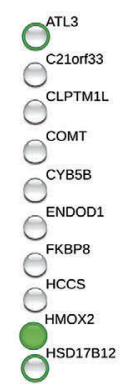

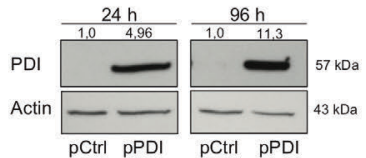

$-\log _{10}$

Probe /

PS89

- 1.5-2.0

$1.0-1.5$

Probe / PS89

E

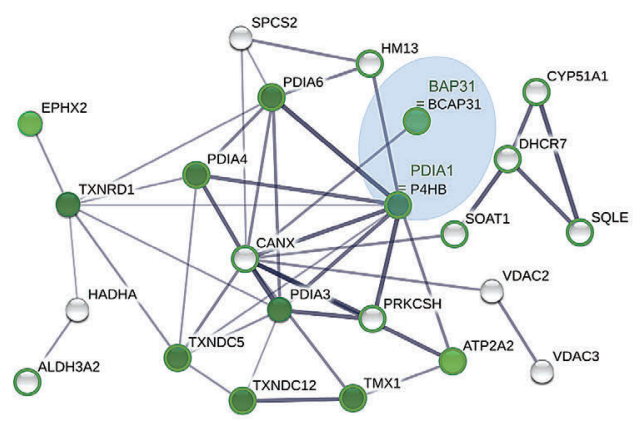

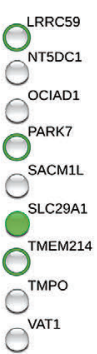

\begin{tabular}{|c|c|c|c|}
\hline Pathway ID & \multicolumn{1}{|c|}{$\begin{array}{c}\text { Pathway } \\
\text { Description } \\
\text { Cellular Component (GO) }\end{array}$} & $\begin{array}{c}\text { Count in } \\
\text { Gene Set }\end{array}$ & FDR \\
\hline GO:0005783 & $\begin{array}{l}\text { Endoplasmic } \\
\text { Reticulum }\end{array}$ & 22 & $5.9 \mathrm{e}-12$ \\
\hline \multicolumn{4}{|c|}{ Biological Process (GO) } \\
\hline GO:0045454 & $\begin{array}{l}\text { Cell Redox } \\
\text { Homeostasis }\end{array}$ & 8 & $6.7 \mathrm{e}-09$ \\
\hline GO:0019725 & $\begin{array}{l}\text { Cellular } \\
\text { Homeostasis }\end{array}$ & 13 & $9.1 \mathrm{e}-07$ \\
\hline
\end{tabular}

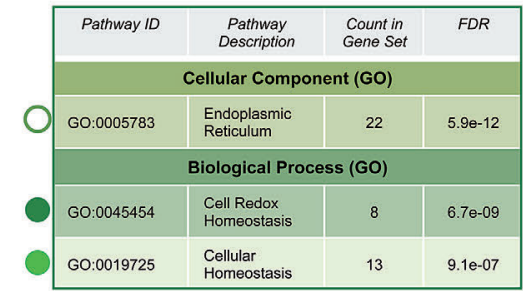

Figure 2. Analysis of PS89 target proteins. (A) Protein disulfide isomerase (PDI)-genetically modified Jurkat cells (siRNA silencing, 48 h) or HEK cells (PDI overexpression, $24 \mathrm{~h}$ ) were treated with etoposide and/or PS 89 for $48 \mathrm{~h}$ and $72 \mathrm{~h}$, respectively. Apoptosis was determined by FACS analysis and inhibition of proliferation by CellTiter-Blue staining. PDI expression was analyzed by immunoblotting, with actin used as a loading control. (B) Principle of activity-based protein profiling with the PS89 photo probe covalently linked to its cellular targets (adapted from Vomacka et $a l_{.^{30}}$ ). (C) Volcano plot of target proteins enriched by the PS89 photo probe versus dimethyl sulfoxide (DMSO) (left). Targets with $>3$-fold enrichment $\left(\log _{2}\right.$ Probe/DMSO $\left.>1.6\right)$ and $-\log _{10} P$-value $>2$ are highlighted. Ranking of targets according to the degree of competition by unmodified PS89 (right). (D,E) Target network analysis of the 42 most enriched PS89-binding proteins with STRING v10. Proteins involved in the most prominent gene ontology classes are highlighted (Cellular Component - green circles; Biological Process - green dots). 
cells with PS89 in combination with etoposide, vincristine or 6-mercaptopurine, respectively, activation of caspase-3 and PARP cleavage indicated a clear induction of apoptotic cell death (Figure 1C, Online Supplementary Figure S2). In addition, apoptosis was prevented by the pan-caspase inhibitor QVD-OPh (Online Supplementary Figure S3). Since pharmacokinetic studies demonstrated that PS89 has a very short half-life in the blood (data not shown), in vivo experiments at not feasible at the moment. However, the broad applicability of PS89 as a chemosensitizing agent was confirmed in ALL and AML PDX cells of diverse backgrounds (Online Supplementary Table S2). PDX samples treated with PS89 and vincristine (Figure 1D) or PS89 and daunorubicin (Figure 1E) showed clearly increased apoptosis rates compared to those treated with the single cytostatics (distinct $P$-values are presented in Online
A

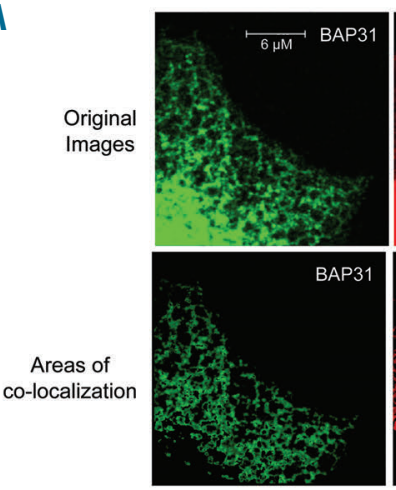

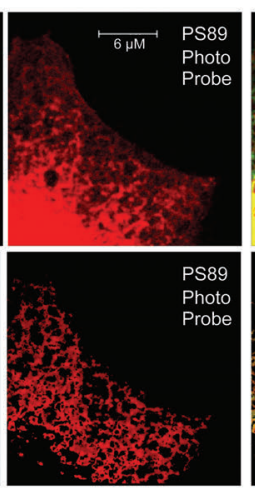

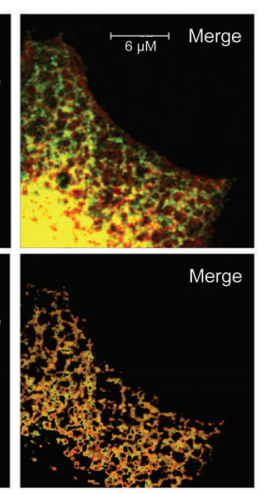

B
50nM PS89 photo probe

50 M PS89 photo probe

+50 nM BAP31 protein

C

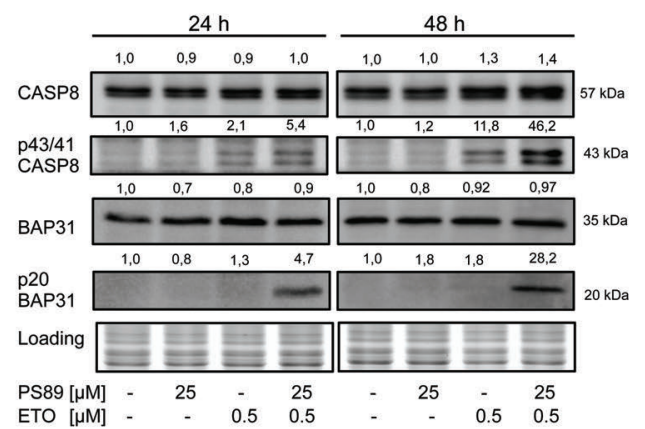

D
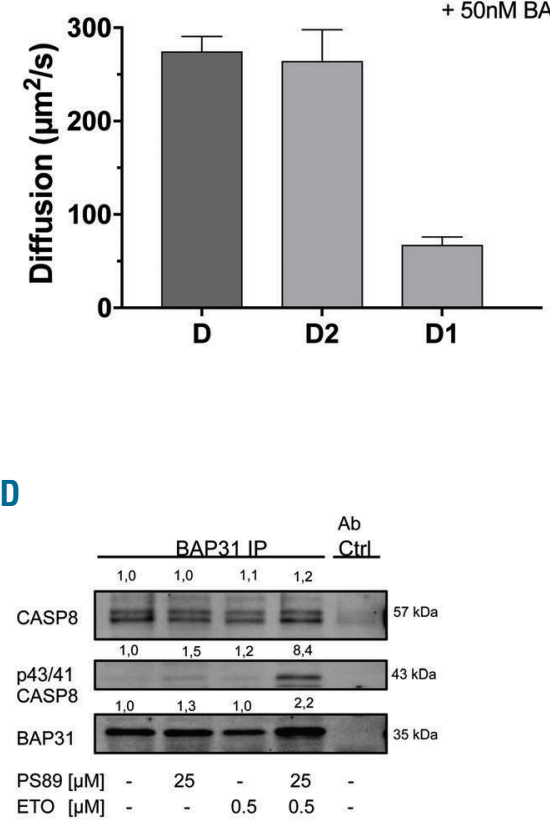

ETO $[\mu \mathrm{M}] \quad-\quad-\quad 0.5 \quad 0.5$

$E$

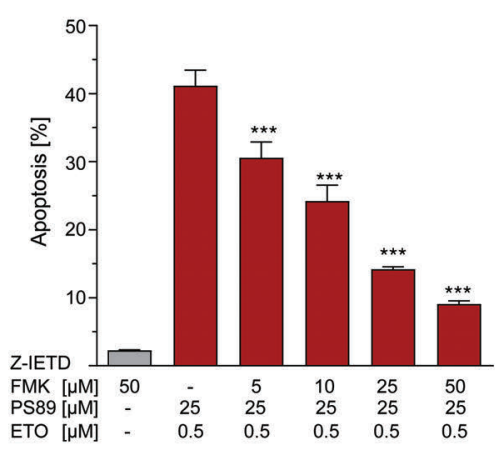

F

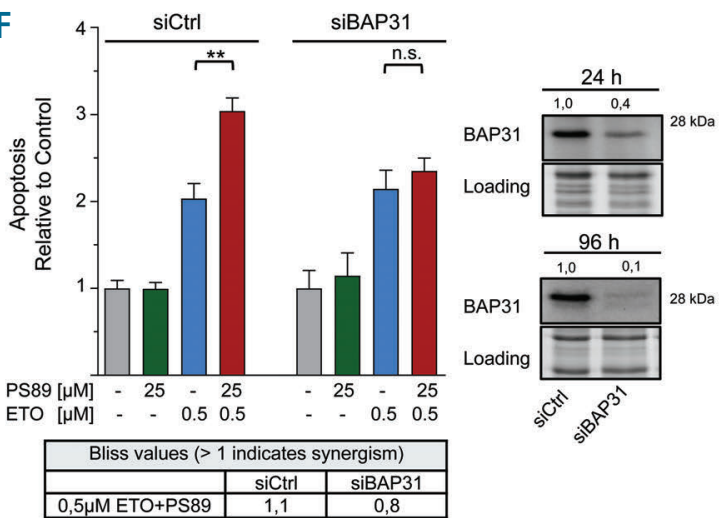

Figure 3. Signal transduction via the BAP31-caspase-8 axis. (A) Immunofluorescence staining of BAP31 (green) and localization of the PS89 photo probe linked to a rhodamine reporter (red) in HeLa cells. Representative original images (upper row) and areas of co-localization analyzed with Leica LAS X software (bottom row) are shown. (B) Diffusion values of PS89 and PS89+BAP31 showing an interaction between the probe and protein. Control samples of $50 \mathrm{nM}$ freely diffusing PS89 in buffer solution and 50 nM PS89 probe plus 50 nM BAP31 protein in buffer solution were analyzed by single-point fluorescence correlation spectroscopy measurements. The diffusion coefficient of the probe alone was measured after one-species fitting of the autocorrelation curves ( $n>15)$. Diffusion coefficients (D1, D2) of S1 and S2 were measured after two-species fitting of the autocorrelation curves ( $n>10)$. The diffusion time (D2) of the mixtures was confined to the diffusion value obtained in the control experiment with PS89 probe alone. Bars represent the mean + standard error of mean. (C) Cleavage of caspase-8 (CASP8) and BAP31 was determined by immunoblotting in Jurkat cells treated with PS89 and etoposide (ETO) for 24 or 48 h. (D) Co-immunoprecipitation of BAP31 and CASP8 from Jurkat cell lysates after $24 \mathrm{~h}$ stimulation with PS89 and ETO. Blots were probed for BAP31 and pro- and intermediate p43/41 CASP8. (E) Apoptosis of Jurkat cells treated with the PS89 and ETO combination in the presence of the specific CASP8 inhibitor Z-IETD-FMK after $48 \mathrm{~h}$. (F) Apoptosis of BAP31-silenced HeLa cells treated for $48 \mathrm{~h}$ with PS89 and ETO ( $6 \mathrm{~h}$ after transfection). The percentage of apoptotic cells was determined by FACS analysis and normalized to controls. The effect of the PS89 + ETO combination versus treatment with ETO alone was analyzed in siCtrl and siBAP31 cells (one-way ANOVA, Tukey test, $P<0.05$ ). 
Supplementary Table $S 3 A, B)$. The Bliss independence model confirmed the synergistic effect of PS89 in combination with vincristine or daunorubicin in ALL and AML PDX cells. Nevertheless, in accordance with previous results, PS89 was non-toxic. Notably, compared to ALL and AML patients' samples, both healthy peripheral blood mononuclear cells and CD34-positive hematopoietic stem cells showed weak responses to combination treatments (Figure 1D-F, Online Supplementary Table S3).
Proteomics identified a PS89 target network affecting endoplasmic reticulum homeostasis

To elucidate the mechanisms underlying the impressive pro-apoptotic effect of PS89 combination treatments, the role of the prominent PS89 target, PDI, was studied by genetic knockdown and overexpression experiments. Since PDI silencing did not mimic and PDI overexpression did not rescue the sensitizing effect of PS89 on apoptosis induction or inhibition on proliferation (Figure $2 \mathrm{~A}$ and

A

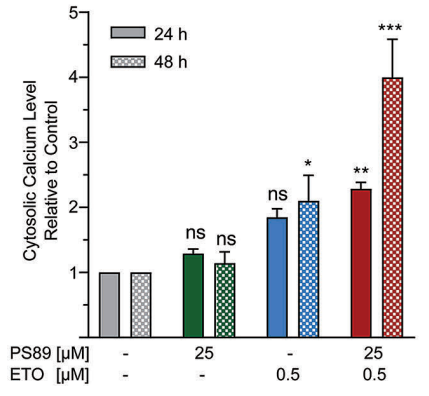

B

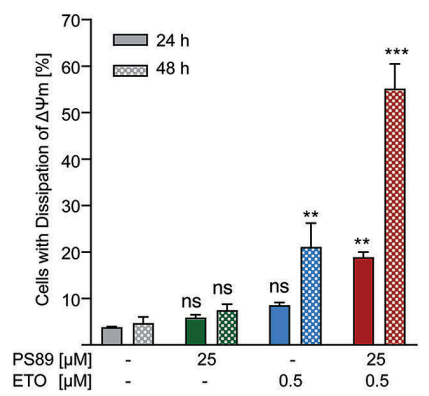

C

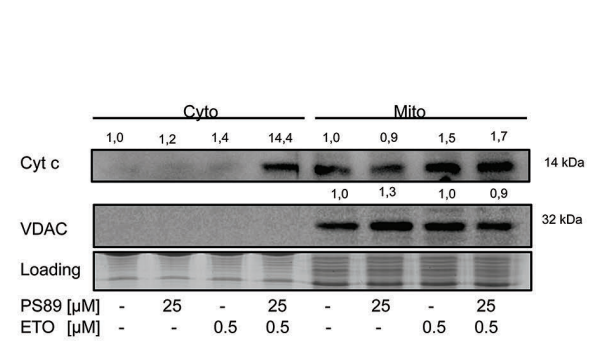

E

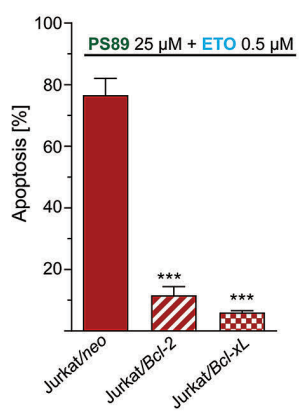

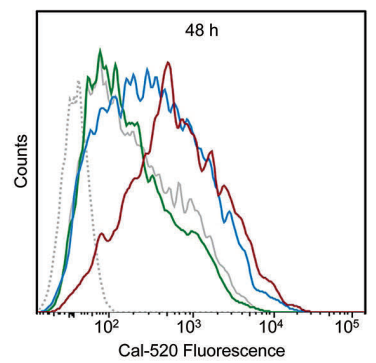

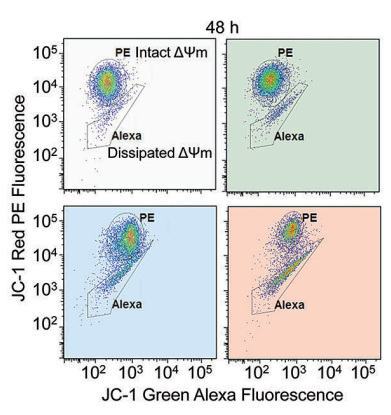

D

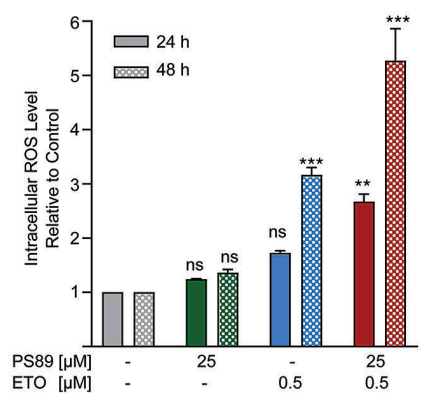

F

Figure 4. Pro-apoptotic crosstalk at the endoplasmic reticulum-mitochondria interface. (A) Intracellular calcium levels of Jurkat cells treated with PS89 and etoposide (ETO) for 24 and 48 h. Fluorescence of Cal-520 stained cells was determined by FACS analysis and mean values normalized towards a dimethyl sulfoxide (DMSO) control. The dotted gray line represents unstained controls. (B) Mitochondrial depolarization of Jurkat cells treated with PS89 and ETO for 24 and $48 \mathrm{~h}$. The percentage of JC-1-stained cells with dissipated versus intact membrane potential $(\Delta \Psi \mathrm{m})$ was determined by FACS analysis (populations as shown by FACS dot plots). (C) Cytochrome c release from mitochondria into the cytosol. Fractionation of Jurkat cell lysates after 48 $\mathrm{h}$ treatment with PS89 and ETO was confirmed by voltage-dependent anion channel (VDAC) immunoblotting Stain-free gels served as the loading control. (D) Intracellular levels of reactive oxygen species (ROS) in Jurkat cells treated with PS89 and ETO for $24 \mathrm{~h}$ and 48 h. Fluorescence of carboxy- $\mathrm{H}_{2}$ DCFDA-stained cells was determined by FACS analysis and mean values normalized to a dimethyl sulfoxide (DMSO) control. The dotted gray line represents unstained controls. (E) Apoptosis of Jurkat vector control (Jurkat/neo), Bcl-2 overexpressing (Jurkat/Bcl-2) and Bcl-xL overexpressing (Jurkat/Bcl-xL) cells treated with ETO and PS89 for $48 \mathrm{~h}$. (F) Apoptosis of Jurkat cells treated with ABT $199(0.5-50 \mu \mathrm{M})$ in the presence or absence of $25 \mu \mathrm{M}$ PS89. The percentage of apoptotic cells was determined by FACS analysis after $48 \mathrm{~h}$ and synergism was calculated using the Bliss independence model. 
Online Supplementary Figure S4), we assumed that PS89 affects additional cellular structures. In order to identify the proposed multi-target characteristics of PS89, we performed activity-based protein profiling in Jurkat cells, as depicted in Figure 2B. The PS89 photo probe modified by an alkyne handle (structure shown in Figure 2B) was covalently linked to cellular targets in the presence or absence of PS89. Co-incubation with the unmodified compound acting as a competitor was performed to exclude the identification of targets that were only enriched by the probe, but not by PS89. Including both datasets as well as their reproducibility expressed as $P$-values (cutoff criteria described in the Methods section), a total of 42 target proteins were identified (Figure 2C and Online Supplementary Table S4). Performing protein-protein interaction analysis using the STRING database, ${ }^{31} 23$ out of the 42 PS89 target proteins were involved in a protein interaction network (Figure 2D). This was further analyzed by gene ontology functional classification for common cellular components and biological processes. ${ }^{32} \mathrm{~A}$ highly significant number of PS89 target proteins was assigned to be located in the ER (false discovery rate $5.9 \times 10^{-12}$ ) and described to be involved in cellular homeostasis, in particular cell redox homeostasis (false discovery rate $9.1 \times 10^{-7}$ and $6.7 \times 10^{-9}$ ) (Figure 2D,E). In this way, BAP31, which is described to be involved in ER stress-mediated apoptosis signaling pathways, ${ }^{15}$ was identified as one of the most prominent target proteins (Figure 2C and Online Supplementary Table S4).

\section{Apoptosis induced by PS89 combination treatments is mediated via the BAP31-caspase- 8 axis}

To validate BAP31 as a direct target of PS89, co-staining was performed with a BAP31-specific antibody and a photo probe linked to a rhodamine reporter dye by click chemistry. Besides the supposed ER specificity, overlap- ping fluorescence revealed distinct co-localized ER network structures of PS89 photo probe-rhodamine and BAP31-Alexa 488 (Figure 3A). No background staining of either the rhodamine-azide or the Alexa 488 secondary antibody was detected (Online Supplementary Figure S5). Direct binding of PS89 photo probe to BAP31 was further evaluated by single-point fluorescence correlation spectroscopy, a technique in which random motion of fluorescent molecules into and out of a stationary laser focus results in fluctuations in fluorescence intensity, which can be monitored by confocal laser scanning microscopy. Hence, diffusion and concentration values of the PS89 photo probe with or without different amounts of recombinant BAP31 protein can be calculated by fitting of the autocorrelation curves (Figure 3B, Online Supplementary Figure S6). As shown in Figure 3B and Online Supplementary Figure S6, whereas the PS89 photo probe alone has a distinct diffusion value of $\sim 274 \mu \mathrm{m}^{2} / \mathrm{s}$, two diffusing components were detected in the presence of recombinant BAP31 protein (D2 and D1). The fast diffusing species D2 characterizes remaining unbound photo probe (showing a diffusion value of $\sim 264 \mu \mathrm{m}^{2} / \mathrm{s}$ ), whereas the slowly diffusing part D1 reflects the PS89 bound to BAP31. This decrease of PS89 diffusion after addition of the BAP31 protein indicates a strong, direct interaction between the two and the resulting diffusion value of $\sim 70 \mu \mathrm{m}^{2} / \mathrm{s}$ is in agreement with literature values for proteins of that size. ${ }^{33}$ Of note, no significant change in concentration was observed after addition of the protein in the solution (Online Supplementary Figure S6D).

As the BAP31 protein complex has been shown to serve as a platform for caspase- 8 activation upon apoptotic stimuli, ${ }^{14}$ the influence of PS89 on caspase- 8 activation after etoposide treatment was examined. Whereas stimulation with etoposide and PS89 alone had only modest

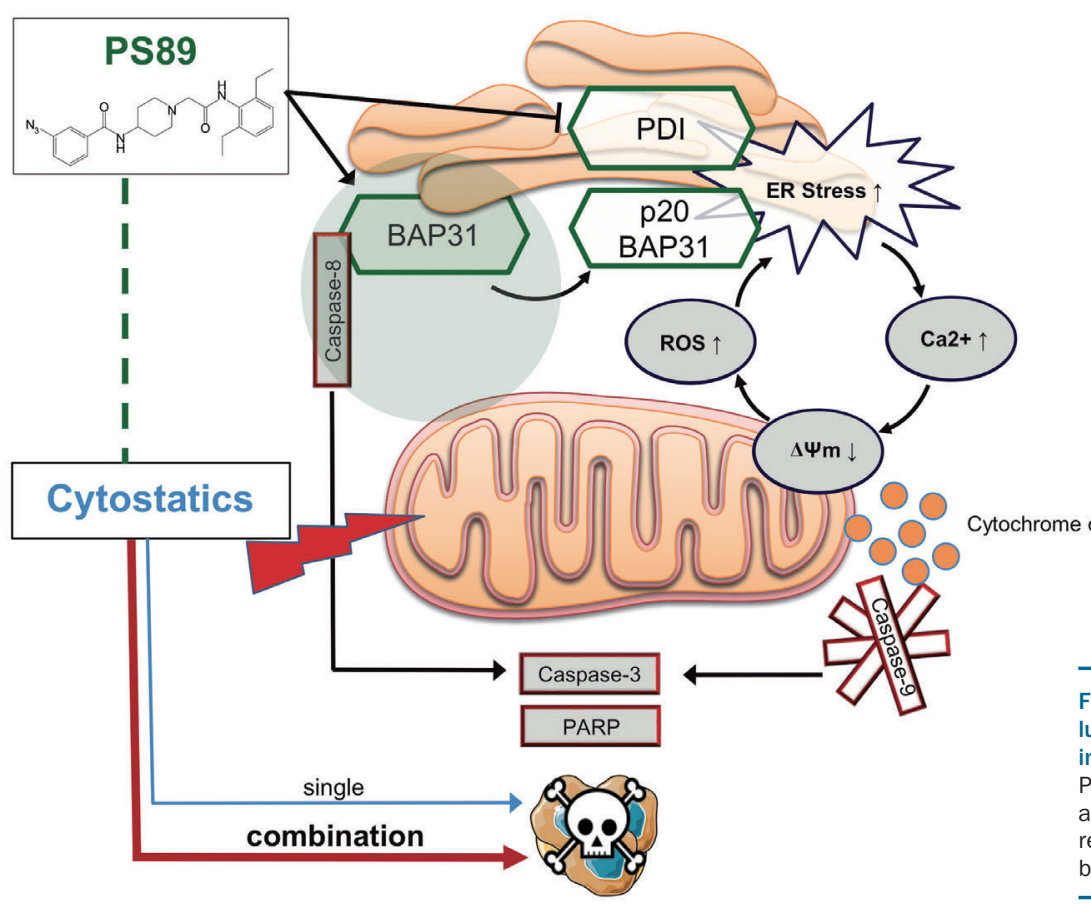

Figure 5. Communication at the endoplasmic reticulum-mitochondria interface in cells treated with PS89 in combination with cytostatics. For details see text. PDI: protein disulfide isomerase; BAP31: B-cell receptorassociated protein 31; ER: endoplasmic reticulum; ROS: reactive oxygen species; $\Delta \Psi \mathrm{m}$ : mitochondrial membrane potential; PARP: poly (ADP-ribosome) polymerase. 
effects on caspase-8 cleavage, the combination of both compounds resulted in strong activation of caspase- 8 in Jurkat, CCRF-CEM as well as ALL PDX samples (Figure 3C, Online Supplementary Figure S7A,B). Moreover, cleavage of BAP31 into the pro-apoptotic p20BAP31 fragment or decreased expression of BAP31 proform was only present in Jurkat, CCRF-CEM and ALL PDX cells treated with the PS89 combination and, as an early trigger, already detectable after $24 \mathrm{~h}$ (Figure 3B, Online Supplementary Figure S7). Treatment-independent interaction of procaspase- 8 with BAP31 could be demonstrated by coimmunoprecipitation (Figure 3D). Interestingly, binding of cleaved p43/p41 caspase-8 to BAP31 was only detected in the presence of both stimulants, PS89 and etoposide (Figure 3D, for normalization to BAP31 see Online Supplementary Figure S8). In order to investigate whether the induction of apoptosis by the combination of PS89 and etoposide is critically dependent on caspase-8 activity, cells were stimulated in the presence of the specific and irreversible caspase-8 inhibitor Z-IETD-FMK. As shown in Figure 3E, inhibiting the activity of caspase-8 resulted in diminished apoptosis upon treatment with etoposide and PS89. In accordance, the ratio of apoptotic cells among the cells treated with the PS89 and etoposide combination versus etoposide alone was reduced in caspase-8-deficient Jurkat cells compared to wildtype cells (1.7-fold in CASP8 $\%$ versus 2.8 fold in wildtype Jurkat cells (Online Supplementary Figure S9). Next, we examined the functional effects of impairing the expression of the direct PS89 target BAP31 by short interfering (si) RNA. Whereas PS89 significantly enhanced etoposide-triggered apoptosis in control cells, no synergistic effect on apoptosis induction upon treatment with PS89 and etoposide could be detected in siBAP31-transfected cells (Figure 3F).

\section{Endoplasmic reticulum and mitochondrial stress triggers are amplified by PS89}

To investigate the consequences of PS89 and etoposide at the ER-mitochondria interface, calcium release from the ER into the cytosol was evaluated by FACS analysis. A shifted Cal 520 fluorescence intensity, indicating higher levels of cytosolic calcium, was observed in Jurkat, CCRFCEM and HL-60 and ALL PDX cells treated with PS89 in combination than in those treated with etoposide alone (Figure 4A, Online Supplementary Figure S10). Interestingly, amplification of calcium release was observed from $24 \mathrm{~h}$ to $48 \mathrm{~h}$ in cells exposed to PS89 combination treatment, but not in cells treated with etoposide, daunorubicin or vincristine alone. Loss of mitochondrial membrane integrity, analyzed by JC-1 staining, was increased by coincubation of etoposide with PS89 (Figure 4B) resulting in release of cytochrome c into the cytosol (Figure 4C, Online Supplementary Figure S11) and elevated levels of reactive oxygen species in Jurkat, CCRF-CEM and HL-60 cells (Figure 4D, Online Supplementary Figure S12). Reactive oxygen species signaling from mitochondria to the ER provokes further disturbance of ER redox homeostasis and finally closes the feedback loop.

The eminent role of functional mitochondrial apoptosis signaling was reinforced by studying stable leukemia cells overexpressing the anti-apoptotic proteins Bcl-2 and Bcl$x L$. Interestingly, these clones showed a significantly lower sensitivity towards PS89 in combination with etoposide than the empty vector cell line Jurkat/neo (Figure $4 \mathrm{E})$. Moreover, specific targeting of mitochondria with the
Bcl-2 inhibitor ABT-199 resulted in synergistic apoptosis in combination with PS89 (Figure 4F, Bliss values are shown in Online Supplementary Table S5), thus substantiating the importance of cytostatic-induced mitochondrial damage provoking the chemosensitizing effect of PS89.

\section{The endoplasmic reticulum-mitochondria interface mediates mutual amplification of PS89 and cytostatic-triggered apoptosis}

In summary, PS89 strongly increases mitochondrial apoptosis through a crosstalk and mutual amplification of pro-apoptotic stress signals triggered by cytostatics (Figure 5). The polypharmacological profile of PS89 affecting a network of ER homeostasis proteins is represented by its main targets PDI and BAP31. Upon apoptotic stimuli of cytostatics and exclusively in the presence of PS89, BAP31 is cleaved by caspase-8 to pro-apoptotic p20BAP31. Calcium release from the ER and increased ER stress promote loss of mitochondrial membrane potential $(\Delta \Psi \mathrm{m})$ and apoptosis. In turn, elevated production of reactive oxygen species feeds back to the ER and provokes further ER stress and calcium release. The mutual amplification of ER-mitochondrial stress triggers finally leads to synergistic activation of caspases and apoptosis.

\section{Discussion}

In the present study, we demonstrated that activating the apoptotic machinery at the ER-mitochondria interface is a highly promising approach for combination drug treatment. Co-stimulation of cytostatics with subtoxic doses of the novel PDI inhibitor PS89 resulted in a highly synergistic apoptotic response in a broad range of ALL and AML cell lines and human xenograft cells derived from both newly diagnosed and relapsed patients. In order to exploit this successful strategy, our work examined the intriguing question of how a drug at non-toxic concentrations could become highly effective in combination with cytostatics.

The small molecule PS89 was previously identified as a potent chemosensitizing agent, which inhibits $\mathrm{PDI}^{22}$ Notably, although PDI plays a key role in the maintenance of oxidative protein folding in the ER, no induction of ER stress or unfolded protein response was observed when applying PS89 alone, but only when the molecule was administered in combination with etoposide. This indicated that activation of the ER stress response results from the disability of the ER to resolve a stressful condition which is provoked in cooperation with cytostatics. ${ }^{22}$

As it is known that many cytostatics induce cell death via activation of the mitochondrial apoptosis pathway ${ }^{34}$ and, moreover, an increasing number of studies indicate a pivotal role of ER-mitochondria communication in cell fate decisions, ${ }^{35-37}$ we investigated a potential PS89-triggered crosstalk between ER stress and mitochondrial damage. Activity-based protein profiling conducted in Jurkat ALL cells identified that, besides PDI, PS89 targets a network of proteins located at the ER, including BAP31. Interestingly, it has been described that etoposide stimulates caspase-8-mediated cleavage of BAP31 to pro-apoptotic p20BAP31 at the ER-mitochondria interface, which results in calcium release from the ER and induction of mitochondrial apoptosis. ${ }^{14}$ Moreover, under conditions of ER stress, BAP31 interacts with cell death-inducing p53- 
target protein 1 (CDIP1) leading to cleavage of BAP31, recruitment of $\mathrm{Bcl}-2$ and mitochondrial apoptosis via Bax oligomerization. ${ }^{15}$ We, therefore, presumed that by inhibiting PDI and further ER stress-related proteins in addition to directly targeting BAP31, PS89 tunes pro-apoptotic feedback from the ER to mitochondria, which results in amplification of cell death signaling in combination therapy.

To confirm the central role of the caspase-8-BAP31 axis in PS89 combination treatment, the processing and activation of the respective proteins was investigated. Indeed, BAP31 cleavage in co-stimulated cells could be observed already at early time points. Interestingly, we were able to show for the first time the intermediate p43/41 cleavage product of caspase- 8 associated with BAP31, which still holds a death effector domain. This supports the suggestion that further processing into the finally active p18 fragment does in fact happen at the BAP31 complex. ${ }^{14}$ As we detected the p43/41 caspase- 8 association as well as BAP31 cleavage only in cells exposed to PS89 combination treatment and silencing of BAP31 rescued the chemosensitizing effect of PS89, we conclude that BAP31 binding is crucial for PS89 to mediate efficient ER-mitochondria communication. Subsequently, an amplification of calcium release was shown in cells exposed to PS89 combination treatment, but not in those treated with etoposide alone. Ultimately, mitochondria-directed calcium flux promotes mitochondrial outer membrane permeabilization, accumulation of reactive oxygen species and release of cytochrome $c, 13,46$ which were demonstrated to be increased in PS89 costimulated cells as well. Hence, PS89 is able to augment apoptotic triggers of cytostatics by interfering with the ER-mitochondria feedback loop. It is noteworthy that cells stably overexpressing the anti-apoptotic mitochondrial proteins Bcl-2 and Bcl-xL are less sensitive to the combination treatment, presumably because of an impaired mitochondrial apoptosis machinery. With reference to the concept of the ER-mitochondria 'social network of cell death', ${ }^{13}$ it is conceivable that the ER-mitochondrial feedback loop procures the crucial pro-apoptotic amplification effect by compromising numerous mitochondria, even if the original stimulus targeted only a few.

In order to further comprehend how stress triggers are communicated from mitochondria to the ER and back, closer examination of the BAP31 complex is required. As shown in previous studies, Fis1 bridges mitochondria and ER-located BAP31 which seems to be further under the control of ER stress-inducible CDIP1 as well as the anti- apoptotic proteins, Bcl-2 and Bcl-xL. ${ }^{14,15}$ However, the dynamics regulating the balance of pro- and anti-apoptotic proteins within the complex have not been clarified yet. As PS89 is, to our knowledge, the first BAP31-binding small-molecule compound, which facilitates BAP31 cleavage, it might serve as a valuable tool not only for studying the dynamics of the BAP31 protein complex and manipulating decisive BAP31 interactions that favor the pro-apoptotic output, but also for enabling in-depth characterization of BAP31 as a prospective pharmacologically addressable target protein in different diseases. With reference to hematologic malignancies, this is further encouraged by the finding that overexpression of BAP31 seems to correlate with chemoresistance, as shown in fludarabine-resistant mantle cell lymphoma ${ }^{38}$ as well as proteasome inhibitor-adapted myeloma cells. ${ }^{39}$

In terms of prospective anti-cancer therapies, targeting pro-apoptotic ER-mitochondria crosstalk by combinatory pharmaceutical intervention offers versatile options. For example, $\mathrm{BH} 3$ mimetics are a valuable novel class of compounds that trigger intrinsic apoptosis and encouraging results were recently shown in an AML phase II trial with ABT-199..$^{40}$ As shown here, ABT-199 in combination with PS89 strongly increases cell death in Jurkat cells compared to the cell death induced by the agents administered singly. This further underlines the concept of amplification by communication between ER and mitochondria as a promising strategy for developing new drugs able to trigger apoptosis and overcome therapy resistance. Moreover, besides PDI-targeting agents, proteasome or HSP90 inhibitors are potentially highly promising candidates for combination with mitochondria-damaging substances to tune the pro-apoptotic ER stress response. ${ }^{41}$

In conclusion and in response to the question of how PS89 is able to sensitize acute leukemia cells, the ER-mitochondria interface was identified as the key platform in the pro-apoptotic signaling cascades mediating the cytotoxic effects of PS89 in combination with cytostatics. By directly affecting PDI and BAP31, PS89 mutually amplifies ER and mitochondrial stress triggers, resulting in strong chemosensitizing effects. Hence, this study reveals the potential of targeting the ER-mitochondria apoptosis network as a novel and encouraging strategy in anti-cancer therapy.

\section{Acknowledgments}

We thank Judith Hoffmann for the supply of PS89 and the photo probe, and Kerstin Loske as well as Silvia Schnegg for technical assistance. The project was financially supported by the Dr. Robert Pfleger foundation.

\section{References}

1. Ward E, DeSantis C, Robbins A, et al. Childhood and adolescent cancer statistics, 2014. CA Cancer J Clin. 2014;64(2):83-103.

2. Pui C-H, Robison LL, Look AT. Acute lymphoblastic leukaemia. Lancet. 2008;371 (9617):1030-1043

3. Döhner $\mathrm{H}$, Weisdorf DJ, Bloomfield CD. Acute myeloid leukemia. New Engl J Med. 2015;373(12):1136-1152

4. Kavallaris M. Microtubules and resistance to tubulin-binding agents. Nat Rev Cancer. 2010;10(3):194-204.

5. Nitiss JL. Targeting DNA topoisomerase II in cancer chemotherapy. Nat Rev Cancer. 2009;9(5):338-350

6. Dieck CL, Tzoneva G, Forouhar F, et al. Structure and mechanisms of NT5C2 mutations driving thiopurine resistance in relapsed lymphoblastic leukemia. Cancer Cell. 2018;34(1):136-147.e6.

7. Nicholson L, Evans CA, Matheson E, et al. Quantitative proteomic analysis reveals maturation as a mechanism underlying glucocorticoid resistance in B lineage ALL and re-sensitization by JNK inhibition. $\mathrm{Br} \mathrm{J}$ Haematol. 2015;171(4):595-605.

8. Jia J, Zhu F, Ma X, et al. Mechanisms of drug combinations: interaction and network per- spectives. Nat Rev Drug Discov. 2009;8(2): 111-128.

9. Barabási A-L, Gulbahce N, Loscalzo J. Network medicine: a network-based approach to human disease. Nat Rev Genet. 2011;12(1):56-68.

10. Zimmermann GR, Lehar J, Keith CT. Multitarget therapeutics: when the whole is greater than the sum of the parts. Drug Discov Today. 2007;12(1):34-42

11. Hopkins AL. Network pharmacology: the next paradigm in drug discovery. Nat Chem Biol. 2008;4(11):682-690.

12. Eletto D, Chevet E, Argon Y, et al. Redox controls UPR to control redox. J Cell Sci. 
2014;127(17):3649-3658.

13. Grimm S. The ER-mitochondria interface: the social network of cell death. Biochim Biophys Acta. 2012;1823(2):327-334

14. Iwasawa R, Mahul-Mellier AL, Datler C, et al. Fis1 and Bap31 bridge the mitochondriaER interface to establish a platform for apoptosis induction. EMBO J. 2011;30(3):556-568.

15. Namba T, Tian F, Chu K, et al. CDIP1-BAP31 complex transduces apoptotic signals from endoplasmic reticulum to mitochondria under endoplasmic reticulum stress. Cell Rep. 2013;5(2):331-339.

16. Rosati E, Sabatini R, Rampino G, et al. Novel targets for endoplasmic reticulum stressinduced apoptosis in B-CLL. Blood. 2010; 116(15):2713-2723.

17. Galligan JJ, Petersen DR. The human protein disulfide isomerase gene family. Hum Genomics. 2012;6:6.

18. Xu S, Sankar S, Neamati N. Protein disulfide isomerase: a promising target for cancer therapy. Drug Discov Today. 2014;19(3): 222-240.

19. Haefliger S, Klebig C, Schaubitzer K, et al. Protein disulfide isomerase blocks CEBPA translation and is up-regulated during the unfolded protein response in AML. Blood. 2011;117(22):5931-5940.

20. Higa A, Taouji S, Lhomond $S$, et al. Endoplasmic reticulum stress-activated transcription factor ATF6 $\alpha$ requires the disulfide isomerase PDIA5 to modulate chemoresistance. Mol Cell Biol. 2014;34(10):1839-1849.

21. Trivedi R, Müller G, Rathore M, et al. Antileukemic activity of shikonin: role of ERP57 in shikonin induced apoptosis in acute myeloid leukemia. Cell Physiol Biochem. 2016:39(2):604-616.

22. Eirich J, Braig S, Schyschka L, et al. A small molecule inhibits protein disulfide isomerase and triggers the chemosensitization of cancer cells. Angew Chem Int ED Engl. 2014;53(47):12960-12965.

23. Xu S, Butkevich AN, Yamada $R$, et al.
Discovery of an orally active small-molecule irreversible inhibitor of protein disulfide isomerase for ovarian cancer treatment. Proc Natl Acad Sci USA. 2012;109(40):1634816353.

24. Vatolin S, Phillips JG, Jha BK, et al. Novel protein disulfide isomerase inhibitor with anticancer activity in multiple myeloma. Cancer Res. 2016;76(11):3340-3350

25. Haber M, Norris MD, Kavallaris M, et al. Atypical multidrug resistance in a therapyinduced drug-resistant human leukemia cell line (LALW-2): resistance to vinca alkaloids independent of P-glycoprotein. Cancer Res. 1989:49(19):5281-5287.

26. Verrills NM, Walsh BJ, Cobon GS, et al. Proteome analysis of vinca alkaloid response and resistance in acute lymphoblastic leukemia reveals novel cytoskeletal alterations. J Biol Chem. 2003;278(46):45082 45093.

27. Terziyska N, Alves CC, Groiss V, et al. In vivo imaging enables high resolution preclinical trials on patients' leukemia cells growing in mice. PLoS One. 2012;7(12): e52798.

28. Vick B, Rothenberg M, Sandhöfer N, et al. An advanced preclinical mouse model for acute myeloid leukemia using patients' cells of various genetic subgroups and in vivo bioluminescence imaging. PLoS One. 2015 10(3):e0120925.

29. Fukuda J, Kaneko T, Egashira M, et al. Direct measurement of CD34+ blood stem cell absolute counts by flow cytometry. Stem Cells. 1998;16(4):294-300.

30. Vomacka J, Korotkov VS, Bauer B, et al. An aromatic hydroxyamide attenuates multiresistant Staphylococcus aureus toxin expression. Chemistry. 2016;22(5):1622-1630.

31. Szklarczyk D, Franceschini A, Wyder S, et al. STRING v10: protein-protein interaction networks, integrated over the tree of life. Nucleic Acids Res. 2015;43(Database issue): D447-452.
32. Consortium GO. Gene ontology consortium: going forward. Nucleic Acids Res. 2015:43(Database issue):D1049-D1056.

33. Krouglova T, Vercammen J, Engelborghs Y. Correct diffusion coefficients of proteins in fluorescence correlation spectroscopy. Application to tubulin oligomers induced by Mg2+ and Paclitaxel. Biophys J. 2004;87(4): 2635-2646.

34. Chonghaile TN, Sarosiek KA, Vo T-T, et al Pretreatment mitochondrial priming correlates with clinical response to cytotoxic chemotherapy. Science. 2011;334(6059): 1129-1133.

35. Bhat TA, Chaudhary AK, Kumar S, et al Endoplasmic reticulum-mediated unfolded protein response and mitochondrial apoptosis in cancer. Biochim Biophys Acta Rev Cancer. 2017;1867(1):58-66.

36. Shore GC, Papa FR, Oakes SA. Signaling cell death from the endoplasmic reticulum stress response. Curr Opin Cell Biol. 2011;23 (2):143-149.

37. Simmen T, Herrera-Cruz MS. Cancer untethering mitochondria from the ER? Front Oncol. 2017;7:105.

38. Lorkova L, Scigelova M, Arrey TN, et al Detailed functional and proteomic characterization of fludarabine resistance in mantle cell lymphoma cells. PLoS One. 2015;10(8):e0135314.

39. Soriano G, Besse L, Li N, et al. Proteasome inhibitor-adapted myeloma cells are largely independent from proteasome activity and show complex proteomic changes, in particular in redox and energy metabolism. Leukemia. 2016;30(11):2198-2207

40. Konopleva M, Pollyea DA, Potluri J, et al. A phase 2 study of ABT-199 (GDC-0199) in patients with acute myelogenous leukemia (AML). Blood. 2014;124(21):118.

41. Wang M, Kaufman RJ. The impact of the endoplasmic reticulum protein-folding environment on cancer development. Nat Rev Cancer. 2014;14(9):581-597. 